\title{
Paper-Based, Inkjet-Printed, Text-Meandered UHF Resonant Antennas for RFID Applications
}

\author{
$\underline{\text { Mauro Marroncelli }}^{1^{*}}$, Daniele Trinchero ${ }^{1+}$, and Manos M. Tentzeris ${ }^{2+}$ \\ ${ }^{1}$ Politecnico di Torino, Corso Duca degli Abruzzi 24, 10129 Torino, Italy; \\ *mauro.marroncelli@polito.it \\ †daniele.trinchero@polito.it \\ ${ }^{2}$ Georgia Institute of Technology, Atlanta, GA 30332, USA; \\ + emmanouil.tentzeris@ece.gatech.edu
}

\begin{abstract}
In this paper different antenna's manufacturing and designing techniques are combined with the aim of building useful radiators resonating in the UHF band. The meander lines techniques is exploited in this work with the exception of letters as meander elements. The technologically advanced and already well established silver inkjet printing technique is used for antenna realization. An unusual paper-based substrate is chosen, according with the concepts to lower costs, simplify fabrication procedures and advance usability, towards the realization of antenna for RFID applications.
\end{abstract}

All design phases are discussed and a complete test of the proposed antennas is performed.

\section{Introduction}

RFID technology has become more and more relevant research field, thanks to few basic characteristics: deployment simplicity, immediate usability, and general low cost. RFIDs have been successfully applied [2] to a large variety of commercial, industrial and leaving environments, e.g. tracking devices, stock management, and biomedical tools for health monitoring. As a natural consequence, important research efforts have been dedicated to make this technology more suitable for a reliable mass production and distribution.

Several aspects must be taken into account when designing antennas for RFID technology. First, the dimensions, due to the fact that a normal RFID device is mainly composed by a transponder and a very simple chipset, where usually the larger space is occupied by the antenna itself. The less the dimension are, the more usable the device will be. Secondly, since the radiator is typically realized with microstrip technology, the choice of the antenna substrate. Materials should be chosen in order to comply the green technology concept. An RFID that is printed on a recyclable substrate, manufactured with standard procedures and utilizing out of the shelf technologies, will undoubtedly increase its appealing to the global industrial market. Finally, to improve its deploying opportunities, the possibility to shape its antenna in ways that would made the antenna itself an object ready for the market commercialization, will clearly conclude its path towards a complete and widely saleable technology device.

The UHF band has nowadays a crucial role for radiofrequency identification, since most of the RIFD applications are set at UHF frequencies. According to [1], the RFID band is allocated on different frequency bands from Country to Country. For example in the US the frequency allocation is set between $902 \mathrm{MHz}$ and $928 \mathrm{MHZ}$, while in Europe is set between 865.6 MHz and 867.6 MHz Designing RFIDs applicable in a large number of Countries it requires developing transponders and antennas able to work on large frequency bands.

In this work we are combining three different technologies to achieve the above stated goal. We are designing an antenna for the UHF band, hence perfectly suitable (providing appropriate tuning) for RFID applications worldwide, which uses a paper-based substrate with inkjet-printed technology for the manufacturing phase, a meander technique for size reduction and a more specific text-based meandered lines to develop an antenna ready for market distribution.

\section{Paper-based, Inkjet-printed Antennas}

The first idea applied in this work is the use of a "green", widely used material such paper as substrate for printed antennas [3]. There are many reason why paper can be considered an efficient solution for RFID's antennas substrate. First, its spread as a common material for thousand of applications, makes it a low-cost, highly flexible, normally available worldwide. Furthermore, from the manufacturing point of view, paper is suitable for mass production, such as direct writing, instead of more traditional etching procedures. Finally, but very important, paper is 
characterized by high biodegradability, which allows it to be considered as environmentally-friendly, in contrast with the normally exploited materials such as generic PCB or FR-4.

Along with the paper substrate, inkjet-printing technique [3] can be used towards the aim of realizing a truly versatile antenna. As a start, by using nozzles to place each single droplet in the wanted position, we can assume a decisive reduction of wasted material, compared to the more common technique of etching, which basically operates a subtractive method removing the unwanted metal from the substrate surface. The ink used in this case is a silver based conductive ink which conductivities varies from $0.4 \sim 2.5 \times 10^{7}$ [Siemens $/ \mathrm{m}$ ], depending on the sintering cycle adopted. More specifically, the sintering process is needed to remove all the impurities from the silver ink and to increase the bonding of the ink to the substrate, by drying out all liquid solvents and particles used to keep the silver liquid during the printing phase.

\section{Meandered Technique}

The concept of meander line antennas (MLA) comes from the simple idea of a folded dipole [3], which is obtained from the antenna itself by bending its own structure. More specifically, a meandered antenna comes from the repetition of the folding principle, meaning that a meandered line it's obtained by the subsequent iterations of the folding principle for a definite amount of times. The final net gain of this technique is to reduce the physical length of the antenna, maintaining the same electrical length [4]. As a matter of fact, it is possible to narrow the total length of the radiator, keeping constant the working frequency of the antenna.

Typically there are two different MLA types, both characterized by definite parameters, such as the horizontal and vertical length of the antenna segments [5]. The first type is the uniform MLA (U-MLA), which is characterized by an equal dimension of the horizontal and vertical segments. The second type is the non-uniform MLA (NU-MLA), which is therefore characterized by a non uniform length of the meandered elements, meaning that the dimensions can vary from one meandered elements to the other. The use of one of the two generally depends on the purpose of the goal to be achieved but, as demonstrated by [5], the main difference in the two elements characterization is the different occupation of space, which is greater for NU-MLAs.

\section{Text-Based Meander Lines}

By folding and bending the radiating elements of an antenna it is possible to reduce the overall physical length, while keeping unchanged the electrical length. This can be done by uniformly bending the antenna or by randomly arranging the meandered elements. Following the latter idea, we could even propose of using letters as an extreme case of meandered line.

As explained in [4], the meander lines derived from this idea are not strictly U-MLA or NU-MLA. This mainly depends on the font and type of chosen text (italics, bold, etc.). The obvious outcome of this technique is the possibility to design the radiating element as a sequence of letters, which can eventually form a word, with an applicable meaning for the manufacturer, or it could even be possible to directly draw logos or brand names or pictures.

\section{Prototyping}

Starting from a normal two-branches dipole structure, and applying the mender process, a text shaped antenna has been developed. The tool used to perform the antenna model was the commercial software HFSS. All the designs can be seen in Fig. 1. The starting point was a dipole design to be resonating at $915 \mathrm{MHz}$ which results in a overall length of $16.39 \mathrm{~cm}$. Folding the antenna by creating a pair of stubs $0.3 \mathrm{~cm}$ in width and $1.7 \mathrm{~cm}$ in vertical length, it was possible to decrease the overall length down to $11.9 \mathrm{~cm}$, thanks to the fact that the stubs modify the reactive part of the antenna impedance allowing a overall horizontal reduction. The successive step was adding more stubs, or even better, applying the text-based meander technique. At the beginning four stubs with letters shape were added, forming the word "love", with dimensions of $0.9 \mathrm{~cm}$ in vertical length and between 0.2 and 0.4 wide, which brings the antenna's length to $12.6 \mathrm{~cm}$. Another important step was the increase of the number of letters, up to six, forming the word "gatech", and analyzing the changes this modification does to the antenna's characteristics. The letters dimension were similar to the one before, in particular they were $0.2 \mathrm{~cm}$ wide and $0.9 \mathrm{~cm}$ tall, and the overall length of the antenna decreased to 11.9 $\mathrm{cm}$. So, as a first consideration, we can assume that by adding more letters" "stub" the total length obviously becomes smaller. 
Al the antenna were consequently printed using the inkjet-printing technique, with silver ink, on paper substrate and finally measured. All the mentioned realizations are shown in Fig. 2.

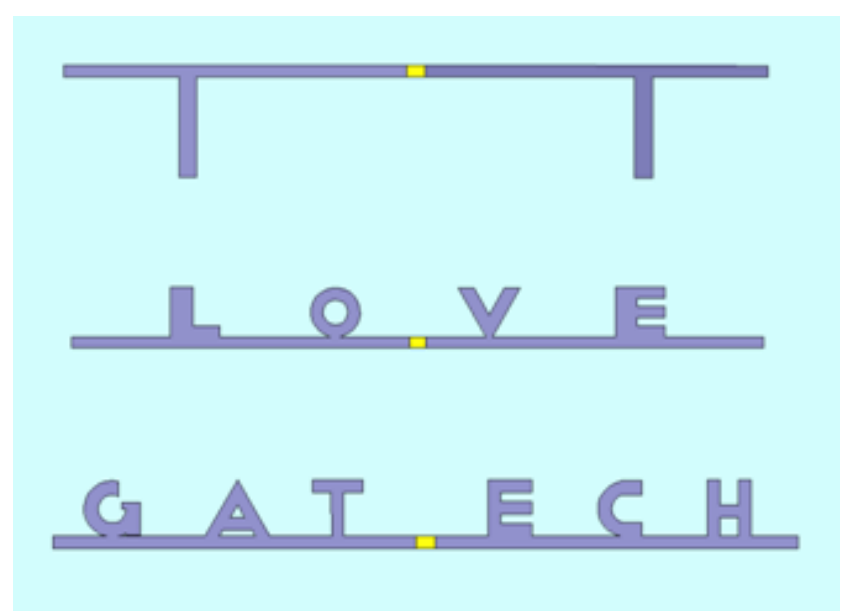

Fig. 1. HFSS' design.

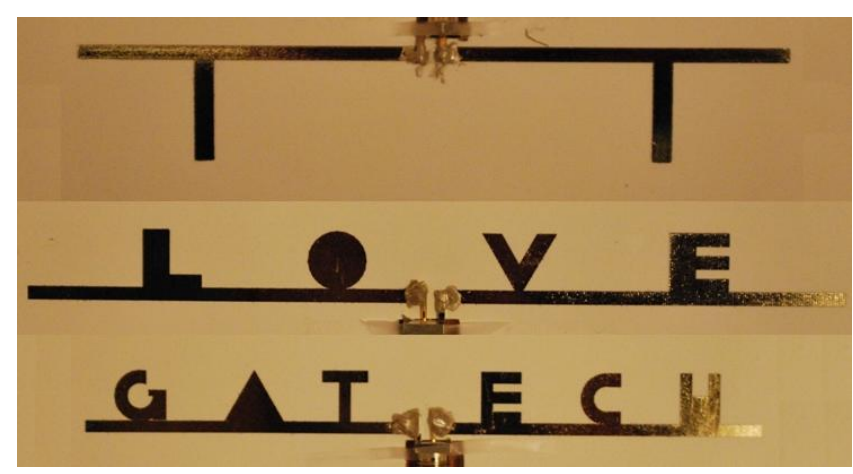

Fig. 2. Silver inkjet-printed antennas.

\section{Measurements}

The last phase of this work was the performance comparison between the simulated antennas and the printed ones. The tool used for this analysis was a R\&S vector network analyzer ${ }^{1}$ for measure the return loss value, with frequency span between $850 \mathrm{MHz}$ and $1.05 \mathrm{GHz}$. Radiation pattern measurements were performed in an anechoic chamber, located at the iXem Labs of Politecnico di Torino. A HP signal generator ${ }^{2}$ able to work in the frequency range between $10 \mathrm{~Hz}$ and $26.5 \mathrm{GHz}$ with a signal strength of $-10 \mathrm{dBm}$ was used. The signal radiated by the antenna was received by a horn antenna with a gain of $6 \mathrm{~dB}$ and send back to a HP spectrum analyzer ${ }^{3}$ working in the frequency range between $30 \mathrm{~Hz}$ and $26.5 \mathrm{GHz}$. All measurements were done in a anechoic chamber and with a movable holder for the transmitting antenna which was able to rotate 360 degrees.

In Fig. 3 we can see the comparison between the simulations results obtained by HFSS and the measurements. Clearly we see a shift of about $30 \mathrm{MHz}$ between the simulated antennas and the real ones. This can be due to a slightly incorrect utilization of the paper parameters in the simulation tools. Comparing measurements to simulations, $\mathrm{S}_{11}$ is slightly lower at the resonating frequency, but this is a normal situation due to the reduced antenna efficiency. Apart from that, we can observe that both the triples of antennas (silver's and HFSS') behave accordingly.

Furthermore, the radiation pattern is similar for all the antennas and can be seen in Fig. 4 . We can observe that the measured radiation pattern matches the one originated from the simulations.

\section{Conclusion}

In this paper we have combined antennas size reduction techniques, with advanced manufacturing techniques, silver-based inkjet printing on paper substrate, towards the aim of building a flexible, low cost and highly usable antennas for the UHF frequency band. Moreover, we have applied the text-based meander lines technique, which use letters as mender elements,.

A careful design followed by a prototyping phase has been carried out, with the result of three different paper based antennas. Finally all the three antennas have been measured and then compared with the simulated results. The comparison shows a good matching and offers interesting future developments

\footnotetext{
${ }^{1}$ ZVB20

${ }^{2}$ HP $863650 \mathrm{~L}$

${ }^{3} \mathrm{HP} 8563 \mathrm{E}$
} 


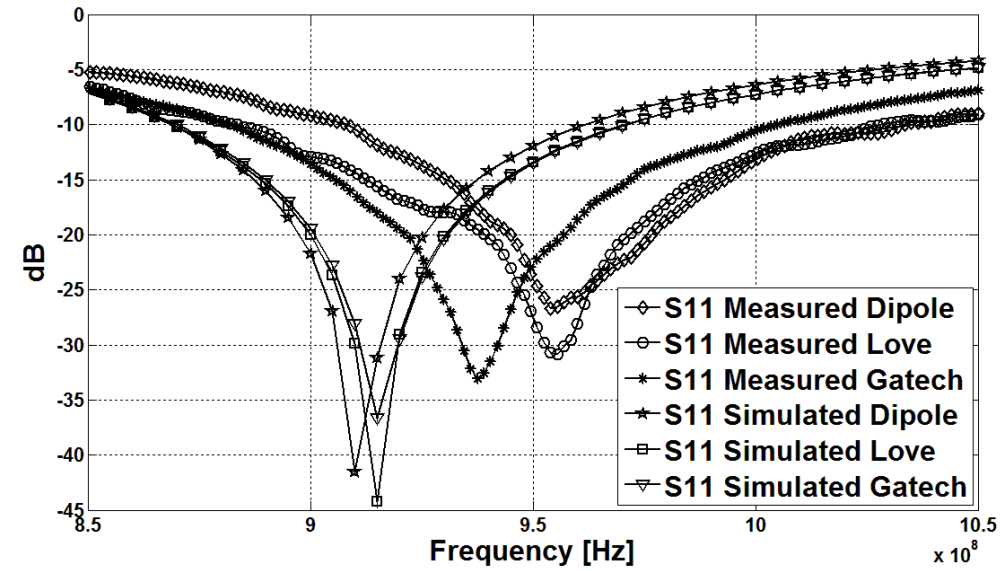

Fig. 3. Comparison between antennas' measured and simulated results.

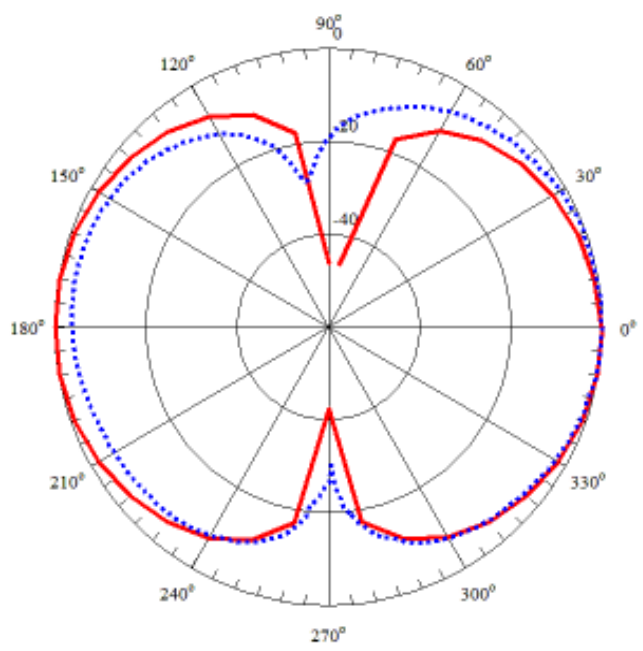

Fig. 4. Radiation pattern.Solid red line simulated, dotted blue line measured.

\section{Acknowledgments}

The authors would like to thank the IFC/SRC Center for their support

\section{References}

1. EPCglobal, Worldwide Frequency Regulatory for RFID, http://www.epcglobalinc.org/tech/.

2. RFID Journal. Online Pubblication. Referenced 2011 at http://www.rfidjournal.com.

3. A. Rida, L. Yang, R. Vyas and M. Tentzeris, "Conductive Inkjet-Printed Antennas on Flexible Low-Cost PaperBased Substrates for RFID and WSN Applications", IEEE Antennas and Propagation Magazine, Vol. 51, No.3, June 2009.

4. C.A. Balanis, Antenna Theory. Analysis and Design, Arizona State University, John \& Sons, 1989.

5. T.J. Warnagiris and T.J. Minardo, "Performance of a Meandered Line as an Electrically Small Transmitting Antenna," IEEE Trans. Antenna Propagat., vol. 46, pp 1797-1876, Dec. 1998.

6. G. Marrocco, "Gain-Optimized Self-Resonant Meander Line Antennas for RFID Applications," IEEE Antennas and Wireless Propagation Letters, Vol. 2, 2003.

7. M. Keskilammi and M. Kivikonski, "Using Text as a Meander Line for RFID Transponder Antennas," IEEE Antennas and Wireless Propagation Letters, Vol. 3, 2004. 